\title{
Téoros
}

Revue de recherche en tourisme

\section{Une table enracinée sur des routes de saveurs}

\section{Lucie Cadieux}

Volume 17, numéro 1, printemps 1998

Le tourisme dans un Pays à part : Charlevoix

URI : https://id.erudit.org/iderudit/1072377ar

DOI : https://doi.org/10.7202/1072377ar

Aller au sommaire du numéro

Éditeur(s)

Université du Québec à Montréal

ISSN

0712-8657 (imprimé)

1923-2705 (numérique)

Découvrir la revue

Citer cet article

Cadieux, L. (1998). Une table enracinée sur des routes de saveurs. Téoros, 17(1), 48-50. https://doi.org/10.7202/1072377ar d'utilisation que vous pouvez consulter en ligne.

https://apropos.erudit.org/fr/usagers/politique-dutilisation/ 


\section{UNE TABLE ENRACINÉE}

\section{SUR DES ROUTES DE SAVEURS}

\section{Lucie Cadieux, présidente de la Table agrotouristique de Charlevoix}

Au caur de la Réserve mondiale de la biosphère, d'où émane une grande richesse du terroir, chefs cuisiniers, producteurs et productrices agricoles de Charlevoix s'allient pour faire connaitre leurs cultures, leurs ellevages et leurs bonnes tables.

Depuis maintenant quatre ans, certaines entreprises agricoles et restaurateurs de Charlevoix offrent un circuit touristique unique en Amérique à tous ceux et celles qui empruntent les routes de Charlevoix; * LA ROUTE DES SAVEURS*. Avant de vous amener avec moi visiter les différentes entreprises du réseau, laissez-moi, vous raconter une histoire: celle de la Table Agro-Touristique de Charlevoix.

\section{PRÉAMBULE}

La Table Agro-Touristique de Charlevoix est un organisme à but non lucratif, légalement fondé en 1994. Elle regroupe des chefs cuisiniers et des producteurs qui entendent travailler ensemble en vue d' offrir soit sur leur table, soit, dans les réseaux de distribution, des produits alimentaires haut de gamme, à forte valeur ajoutée. Cette table s'est élargie depuis 1994 avec l'implication à la fois des représentants des deux UPA (Union des producteurs agricoles), d'un représentant du MAPAQ (Ministère de l'agriculture, des pêcheries et de l'alimentation), des deux commissaires au dév veloppement ểconomique et de transformateurs.

Les mandats de la Table Agro-Touristique de Charlevoix ont été définis comme étant ceux de développer et promouvoir les produits régionaux haut de gamme et de garantir la qualité et la spécificité de ces derniers par la mise en place de signes de qualites reconnus de types IGP (Identification géographique protégée), Labels ou AOC (Appellation d'origine contrôlée).

La mission de la Table Agro-Touristique de Charlevoix est la suivante:

\begin{abstract}
- Dynamiser le tissu social et agroalimentaire de Charlevoix en favorisant le développement d'un pro. cessus d'intégration des phases de production, de transformation et de commercialisation des produits du terroir afin d'offrir des produits de qualité unique w.
\end{abstract}

La Table Agro-Touristique de Charlevoix permet de créer des liens entre producteurs et chefs de la région. Leur dynamisme et leur volonté de se démarquer en offrant des produits de qualité sont des éléments culture de Charlevoix.

\section{LA ROUTE DES SAVEURS}

La Route des saveurs, est une initiative du comité de commercialisation de la Table Agro-Touristique de Charlevoix. Elle permet a la population locale et touristique de faire le lien entre la production et les produits. Le visiteur qui cherche moteurs dans le développement de l'agri-

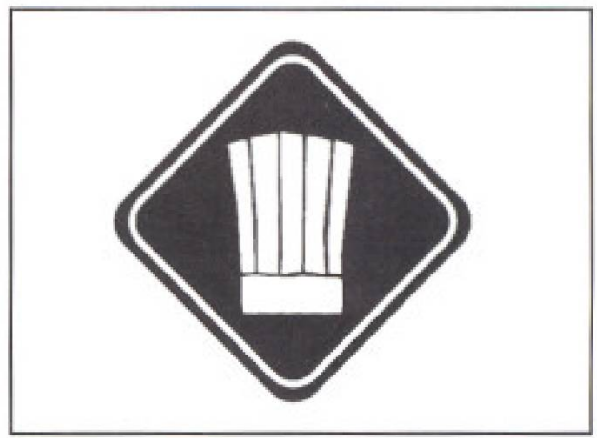

à découvrir les beautés visuelles de Charlevoix peut aussi se familiariser avec les saveurs spécifiques de la région. Dans les auberges, les menus identifient les matieres premiêres issues de la région. Personnels de service et chefs parlent de produits du terroir et invitent les gens à visiter les entreprises qui les produisent.

Ce concept unique au monde (mariage entre chefs et entreprises agricoles) met en exergue les produits du terroir et permet de créer un lien sans précédent avec les consommateurs par le biais du talent incontesté de nos chefs cuisiniers. Les producteurs et productrices agricoles participant à la Route des saveurs distribuent directement aux auberges et restaurants de la région leurs produits (légumes, veaux, agneaux, petits fruits, truites, fromage etc.) ou vendent à la ferme.

\section{QU'EN ESTIIL AU JUSTE ?}

Les membres de la Route des saveurs sont identifiés par un panneau signalétique rose, représentant lạ toque des Chefs. En 1997, neuf entreprises agricoles, cinq transformateurs et treize auberges et restaurants étaient membres du circuit de la 
Route des saveurs. Partout, les touristes sont accueillis avec diligence et les producteurs et productrices se font un devoir de vous expliquer leurs méthodes de production, d'élevage ainsi que les exigences des chefs face à leurs produits. De même, si on va déguster un repas dans une des auberges membre de la Route des saveurs, ou y trouve des gens en mesure de vous parler des produits qu'ils utilisent dans la composition de leur menu Route des saveurs. Bien plus, ils vous inviteront à prendre la Roule des saveurs.

Il est à noter que les membres font l'objet d'un contrôle serré́ tant du côté des producteurs et transformateurs (qualité de la visite, accueil, propreté des lieux et qualité de l'information diffusée) que du côté des restaurants et auberges (obligation d'un plat principal avec des produits de la région, vérification des factures d'achats de produits de Charlevoix).

\section{PRENEZ LA ROUTE DES SAVEURS}

Pour avoir le meilleur aperçu possible de l'état actuel de toute l'entreprise, aussi bien prendre la route et suivre le guide.

\section{CHEZ LES PRODUCTEURS}

En suivant le panneau signalétique rose, orné d'une toque de chefs, vous découvrirez d'où viennent les produits régionaux qui se retrouvent dans les assiettes des auberges et des restaurants de Charlevoix. Cette grande virée vous amènera dans tous les terroirs charlevoisiens. Débutons notre visite par la Maison d'affinage Maurice Dufour. M. Dufour vous expliquera comment est né le célèbre fromage Le Migneron et pourquoi il est maintenant reconnu comme un des meilleurs fromages au Canada. Vous pourrez aussi goûter à ce fromage fait du lait de troupeaux de vaches de la vallée de Baie-St-Paul et selon des critères de selection ếtablis en partenariat avec l'affineur et les producteurs. Pourquoi ne pas aussi profiter de votre passage a Baie-St-Paul pour aller visiter l'économusce de la Laiterie Charlevoix où vous pourrez y voir les différentes étapes de transformation du lait en fromage?

En quittant Baie-St-Paul et en empruntant la route panoramique qui longe le majes-

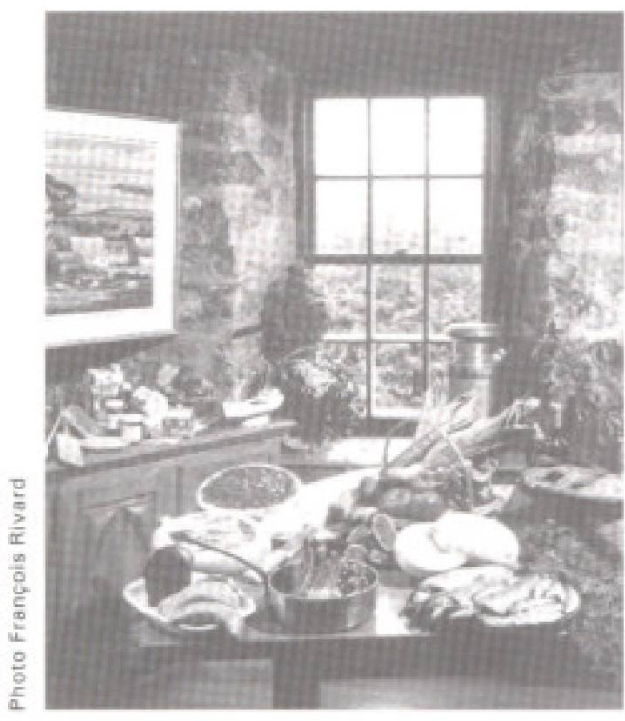

Revenu aux Éboulements, lournez à droile, en direction de Saint-Irénée. Tout juste à la sortie du village, arrêtez-vous à La Ferme Eboulmontaise. Les propriétaires et leurs enfants vous feront découvrir toutes les facettes de l'élevage de l'agneau de Charlevoix, ainsi que la culture des légumes biologiques. Vous aurez l'opportunité de visiter le centre d'interprétation de la bergerie et de terminer votre visite au kiosque de vente. Pourquoi ne pas en profiter pour vous y procurer un gigot d'agneau, quelques côtelettes, des légumes et des fines herbes afin de vous concocter un repas d'amoureux. Ah oui! Un petit bouquet de fleurs fraichement coupées agrémentera votre table.

A La Malbaie, allez rencontrer Hervé Gobeil à la boulangerie Le Temps d'un Pain. Il saura vous expliquer comment il a fabriqué ses fours à pain selon la plus pure tradition d'Alexis Le Trotteur. Vous repartirez sürement de la boulangerie les bras chargés de pains, baguettes ou tartes. acheter de (nature ou fumée) ou de l'onctueuse mousse de truite.

Après cette visite, arrêtez-vous au Moulin Seigneurial des Éboulements. Le meunier vous mettra dans le secret des dieux et vous fera visiter un moulin tout à fait exceptionnel construit en 1790 . La mouture du blé et du sarrasin s'y fait selon la méthode ancestrale.

En poursuivant votre route, n oubliez pas de faire une halte aux Jardins du Centre (en direction de Saint-Joseph-de-laRive) où toute une équipe de gens vous attend pour vous faire visiter les jardins et le centre de transformation. Le décor vous coupera le souffle. Vous pourrez vous y procurer des légumes particuliers tels la betterave jaune, la bette à carde, la fameuse gourgane et tous les légumes qui agrémentent si bien un bon repas. N'oubliez surtout pas de prendre un pot de confiture d'oignon, extra!

Comme vous êtes à mi-chemin entre les Eboulements et Saint-Joseph de la Rive, allez prendre le bateau pour l'île aux Coudres. Une fois rendu chez les insulaires, dirigez-vous vers les Vergers Pedneault. Vous pourrez y cueillir des pommes et déguster les produits transformés de la pomme: le jus, les gelées, les quartiers de pommes dans le sirop ou encore ce délicieux beurre de pomme.
La route 138 vous amène cnsuite dans l'arrière-pays. Allez rendre visite à $\mathrm{M}$. Martel (La Vallée des cervidés est située A la Rivière Malbaie) pour vous familiariser avec l'élevage de cerf rouge. Vous pourrez y visiter les enclos et les bâtiments où y sont élevés les chevreuils. Après avoir discuté avec M. Martel. il vous invitera surement à vous rendre chez son ami $\mathrm{M}$. Ghislain Bilodeau (La Ferme d'élevage des sangliers de Saint-Aimé-des-Lacs) qui élève des bisons et des sangliers. Sur place vous pourrez vous promener en forêt et observer les sangliers et leurs marcassins ainsi que de fabuleux bisons qui font la fierté de l'éleveur. Il vous aura certainement préparé un léger goûter avec des produits à base de sanglier: terrines, pâtếs et autres délices.

Dans le Parc des Grands Jardins, faites une halte à la Réserve charlevoisienne des cervidés ou Martin et Michel vous permettront d'observer les daims dans leur habitat naturel. Ils vous expliqueront aussi comment sont manipulés et identifiés les animaux. Vous pourrez aussi nourrir les daims. Avant de poursuivre votre chemin, vous pourrez vous procurer du Veau saveur au centre de transformation et d"abattage Le Veau de Charlevoix. situé à Clermont. Jean-Robert Audet vous renseignera sur les différentes méthodes d'abattage et de transformation des ani- 
maux. II discutera certainement avec vous de la vision qu"il a de la transformation et de la commercialisation des produits carnés de la région, Sur le chemin du retour, prenez une pause à la Ferme des Marais. Diane et Jean vous entretiendront de leur nouvelle passion, l'émeu et le nandou. Vous n'y connaissez rien à ces volatilles? qu'd cela ne tienne, vous profitez des connaissances de vos deux experts.

Essoufflé! La tournée des entreprises agricoles est terminée. II vous reste à aller saluer Herman à la Sucrerie Herman Bouchard de Petite-Rivière-Saint-François, un maître sucrier qui vous dévoilera presque tous ses petits secrets qui sucrent le bec.

\section{CHEZ LES AUBERGES ET RESTAURANTS}

Après le tour des entreprises agricoles, il vous reste à passer à table. Vous aurez l'embarras du choix. Treize auberges et restaurants se trouvent sur la Route des saveurs. Ils vous offriront un menu composé de produits frais de la région et des plats cuisinés Route des saveurs. Vous pourrez donc déguster cette fine cuisine

\begin{tabular}{|c|c|c|}
\hline ENDROIT & ÉTABLISSEMENT & CHEF \\
\hline Petite-Rivière-St-François & Le Massif de Petite-Rivière-St-François & M. Guy Bessone \\
\hline Baie-St-Paul & $\begin{array}{l}\text { Restaurant Le Mouton Nair } \\
\text { Restaurant La Muse } \\
\text { Auberge La Maison Dtis }\end{array}$ & $\begin{array}{l}\text { M. Guy Bessone } \\
\text { Mme Evelyne Tremblay } \\
\text { M. Bernard Tapin }\end{array}$ \\
\hline St-Joseph-de-la-Rive & Auberge Motel Beausejour & Mme Diane Dufour \\
\hline St-Irénẻe & Auberge des Sablons & M. Rlobert Jardin \\
\hline Pointe-Au-Pic & $\begin{array}{l}\text { Manoir Richelieu } \\
\text { Auberge des Trois Canards } \\
\text { Auberge des Falaises } \\
\text { Auberge La Petite Marmite } \\
\text { Auberge restaurant Sur La Côte }\end{array}$ & $\begin{array}{l}\text { M. Henry Meesen } \\
\text { M. Eric Bertrand } \\
\text { M. Régis Hervé } \\
\text { M. Guy Thibodeau } \\
\text { M. Jean-Louis Maltais }\end{array}$ \\
\hline Cap-A-L'Aigle & Auberge des Peulpiers & M. Dominique Truchon \\
\hline St-Aimé-des-Lacs & Auberge Le Relais des Hautes-Gorges & M. Rhèal Séguin \\
\hline
\end{tabular}

tout en vous remémorant les bons moments passés en compagnie des producteurs et productrices que vous aurez prélablement visités au cours de votre sejour dans Charlevoix.

Que vous soyez à Baie-Saint-Paul, a la Petite-Rivière-Saint-François, dans le parc des Hautes-Gorges, à Saint-Joseph-de-la Rive, Saint-Irénée ou encore à La Malbaie, vous y serez reçu, vous et votre famille, dans la plus pure tradition d'hospitalité des gens de Charlevoix.
Voici la liste (voir tableau), des auberges et des restaurants qui sont membres de la Route des saveurs.

La visite est terminée, alor's, si nous avons réussi à vous donner le goût de venir nous rencontrer, il nous fera un immense plaisir de vous accueillir dans nos entreprises agricoles où dans nos auberges et achouenners avec vous.

\section{TÉOROS VOUS PRÉSENTE SA COLLECTION COLLOQUES ET CONGRES}

\section{UN AUTRE TRAIT D'UNION ENTRE LE MONDE DE LA RECHERCHE ET CELUI DES PROFESSIONNELS}

Cette collection réunit les textes des conférences et des communications prononcées lors de différents colloques nationaux et internationaux qui ont eu lieu au Québec depuis 1991.

Ainsi, chaque numéro vous permet de connaître, sur un sujet donné, ce qui se dit et ce qui se fait ici et ailleurs... et ce que des specialistes et des intervenants de divers horizons et de diverses régions en pensent...

\section{Deja parus}

No 1: Les politiques touristiques (34 p. + cartes)

No 2: Premier colloque national sur le tourisme de circuit (44 p.)

No 3: Colloque international uFleuves et planèten (52 p.)

No 4: Colloque national sur l'écotourisme (58 p.)

No 5: Colloque wTourisme et culturew, une alliance d'avenir? (48 p.)

No 6: Premier colloque national sur le golf $(46 \mathrm{p}$.

No 7: Troisième colloque «École sans frontièresı Le tourisme éducatif et les besoins des ainès $(200 \mathrm{p}$-)

No 8: Colloque sur l'écotourisme (0uest du Québec) (68 p.)

No 9: Colloque québécois sur le tourisme d"aventure: 1996 l'année de tous les risques (68 p.)

No 10: Premier congrès de la Fédération québécoise des offices du tourisme (F0OT) (44 p.)

No 11: Premier colloque national sur l'agrotourisme (51 p.)

No 12: Deuxième colloque national sur l'agrotourisme (à venir) Les prix incluent les taxes

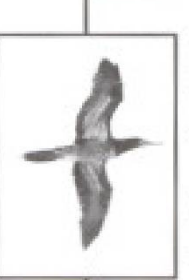

\section{$9 s$}

9 S $9 \mathrm{~s}$ 95 $9 \mathrm{~s}$ gs $40 s$ 9s 9 $9 \mathrm{~s}$ $9 \mathrm{~s}$ 95

9s
- Statistiques: regroupe les chroniques aconjonctures,
déjă publiées, sur les clientèles
touristiques du Québec:
DU NOUVEAU SUR LE SITE INTERNET DE TÉOROS hitp:/www.unites.uqam.ca/teoros/
Voila maintenant quelques mois que vous avez accès, au bout du fil, à une foule d'informations sur la revue.

Le site Internet de Téoros inaugure trois nouvelles façons de vous informer sur la recherche en tourisme dans le monde francophone.

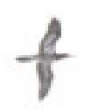

